\title{
MOLECULAR GAS IN HICKSON COMPACT GROUPS
}

\author{
S. LEON AND F. COMBES \\ DEMIRM, Observatoire de Paris, France
}

AND

T.K. MENON

Dep. of Physics \& Astronomy, Univ. of British Columbia, Vancouver, Canada

Compact groups are ideal sites to study the influence of strong dynamical evolution due to environment on molecular cloud formation and star formation efficiency. We have observed 70 galaxies belonging to 45 Hickson compact groups (HCGs) in the ${ }^{12} \mathrm{CO}(1 \rightarrow 0)$ and ${ }^{12} \mathrm{CO}(2 \rightarrow 1)$ lines, in order to determine their molecular content. We compare the gas content relative to blue and $L_{\mathrm{FIR}}$ luminosities of galaxies in compact groups with respect to other samples in the literature, including various environments and morphological types. We find that there is some hint, of enhanced $M_{\mathrm{H}_{2}} / L_{\mathrm{B}}$ and $M_{\text {dust }} / L_{\mathrm{B}}$ ratios in the galaxies from compact group with respect to our control sample, especially for the most compact groups, suggesting that tidal interactions can drive the gas component inwards, by removing its angular momentum, and concentrating it in the dense central regions, where it is easily detected. The threshold at $20-30 \mathrm{kpc}$ in mean galaxy separation for the enhancement of $\mathrm{H}_{2}$ suggests that it must correspond to an acceleration of the merging process and a significant inward gas flow. The molecular gas content in compact group galaxies is similar to that in pairs and starburst samples. However, the total $L_{\text {FIR }}$ luminosity of HCGs is quite similar to that of the control sample, and therefore the star formation efficiency appears lower than in the control galaxies. However this assumes that the FIR spatial distributions are similar in both samples which is not the case at radio frequencies. Higher spatial resolution FIR data are needed to make a valid comparison. Given their short dynamical friction time-scale, it is possible that some of these systems are in the final stage before merging, leading to ultraluminous starburst phases. We also find for all galaxy samples that the $\mathrm{H}_{2}$ content (normalized to blue luminosity) is strongly correlated with $L_{\mathrm{FIR}}$, while the total gas content $\left(\mathrm{H}_{2}+\mathrm{HI}\right)$ is not. 